OPEN ACCESS

\section{The PIENU experiment at TRIUMF : a sensitive probe for new physics}

To cite this article: Chloé Malbrunot et al 2011 J. Phys.: Conf. Ser. 312102010

View the article online for updates and enhancements.
Related content

- Status of the PIENU experiment

Experimental study of rare charged pion decays

Beyond the Standard Model with Electron and Muon Beams

\section{Recent citations}

- Beta decavs and non-standard
$\frac{\text { interactions in the LHC era }}{\text { Vincenzo Cirigliano et al }}$
- FCNC portals to the dark sector
Jernej F. Kamenik and Christopher Smith 


\title{
The PIENU experiment at TRIUMF : a sensitive probe for new physics
}

\author{
Chloé Malbrunot ${ }^{1,2}$, A A Aguilar-Arevalo ${ }^{3}$, M Aoki $^{4}$, M Blecher ${ }^{5}$, \\ D I Britton ${ }^{6}$, D A Bryman ${ }^{1,2}$, S Chen ${ }^{7}$, J Comfort ${ }^{8}, \mathbf{M}$ Ding $^{7}$, \\ J Doornbos ${ }^{2}$, L Doria ${ }^{2}$, P Gumplinger ${ }^{2}$, C Hurst ${ }^{1}$,

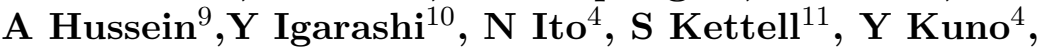 \\ L Kurchaninov ${ }^{2}$, L Littenberg ${ }^{11}$, T Numao ${ }^{2}$, R Poutissou ${ }^{2}$, A Sher ${ }^{2}$, \\ T Sullivan ${ }^{1}$, D Vavilov ${ }^{2}, \mathrm{~K}$ Yamada $^{4}, \mathbf{M}$ Yoshida $^{10}$ \\ ${ }^{1}$ Dep. of Physics and Astronomy, University of British Columbia, B.C. , Canada \\ 2 TRIUMF, 4004 Wesbrook Mall, Vancouver, B.C. Canada \\ ${ }^{3}$ Instituto de Ciencias Nucleares, U.N.A.M, Mexico City, Mexico \\ ${ }^{4}$ Physics Dep., Osaka University, Toyonaka, Osaka, Japan \\ ${ }^{5}$ Physics Dep., Virginia Tech., Blacksburg, VA, USA \\ ${ }^{6}$ Dep. of Physics and Astronomy, University of Glasgow, Glasgow, UK \\ 7 Physics Dep., Tsinghua University, Beijing, P. R. China \\ ${ }^{8}$ Physics Dep., Arizona State University, Tempe, AZ USA \\ ${ }^{9}$ Physics Dep., Northern University of British Columbia, Prince George, B.C., Canada \\ ${ }^{10}$ KEK, 1-1 Oho, Tsukuba-shi, Ibaraki-ken, Japan \\ ${ }^{11}$ Brookhaven National Laboratory, Upton, NY, USA \\ E-mail: chloe@triumf.ca
}

\begin{abstract}
Study of rare decays is an important approach for exploring physics beyond the Standard Model (SM). The branching ratio of the helicity suppressed pion decays, $R=$ $\frac{\Gamma\left(\pi^{+} \rightarrow e^{+} \nu_{e}+\pi^{+} \rightarrow e^{+} \nu_{e} \gamma\right)}{\Gamma\left(\pi^{+} \rightarrow \mu^{+} \nu_{\mu}+\pi^{+} \rightarrow \mu^{+} \nu_{\mu} \gamma\right)}$, is one of the most accurately calculated decay process involving hadrons and has so far provided the most stringent test of the hypothesis of electron-muon universality in weak interactions. The branching ratio has been calculated in the SM to better than $0.01 \%$ accuracy to be $\mathrm{R}_{S M}=1.2353(1) \times 10^{4}$. The PIENU experiment at TRIUMF, which started taking physics data in September 2009, aims to reach an accuracy five times better than the previous experiments, so as to confront the theoretical calculation at the level of $\pm 0.1 \%$. If a deviation from the $R_{S M}$ is found, "new physics" beyond the SM, at potentially very high mass scales (up to $1000 \mathrm{TeV}$ ), could be revealed. Alternatively, sensitive constraints on hypotheses can be obtained for interactions involving pseudoscalar or scalar interactions. So far, 4 million $\pi^{+} \rightarrow e^{+} \nu_{e}$ events have been accumulated by PIENU. This paper will outline the physics motivations, describe the apparatus and techniques designed to achieve high precision and present the latest results.
\end{abstract}

\section{Introduction}

The branching ratio of pion decays, $R=\frac{\Gamma\left(\pi^{+} \rightarrow e^{+} \nu_{e}+\pi^{+} \rightarrow e^{+} \nu_{e} \gamma\right)}{\Gamma\left(\pi^{+} \rightarrow \mu^{+} \nu_{\mu}+\pi^{+} \rightarrow \mu^{+} \nu_{\mu} \gamma\right)}$ has provided the best test of the hypothesis of electron-muon universality in weak interactions. The most recent experimental results of the branching ratio are: $R=(1.2265 \pm 0.0034$ (stat) \pm 0.0044 (syst) $) \times 10^{-4}$ (TRIUMF) [1] and $R=(1.2346 \pm 0.0035$ (stat) \pm 0.0036 (syst) $) \times 10^{-4}$ (PSI) [2]. The new TRIUMF PIENU 
experiment aims to improve the precision of the branching ratio measurement by a factor of $>5$, confronting the Standard Model $(\mathrm{SM})$ prediction of $R_{S M}=1.2352(1) \times 10^{-4}[3,4]$ to better than $0.1 \%$. At that level, new physics may be heralded by a deviation from the precise SM expectation. In particular, it is very sensitive to helicity unsuppressed decays involving a pseudo-scalar. Because any pseudo-scalar contribution comes as an interference with the dominant axial-vector interaction, the contribution is proportional to $1 / \mathrm{m}_{\Lambda}^{2}$, where $\mathrm{m}_{\Lambda}$ is the mass of a hypothetical particle. Ignoring small contributions from $\pi \rightarrow \mu \nu$ decay in the presence of pseudoscalar interactions and assuming a coupling similar to the weak coupling, the deviation of the new branching ratio from the SM prediction can be parameterized as [5]

$$
1-\frac{R_{e / \nu}^{E x p}}{R_{e / \nu}^{S M}} \sim \pm \frac{\sqrt{2 \pi}}{G_{\mu}} \frac{1}{\Lambda_{e P}^{2}} \frac{m_{\pi}^{2}}{m_{e}\left(m_{d}+m_{u}\right)} \sim\left(\frac{1 T e V}{\Lambda_{e P}}\right)^{2} \times 10^{3}
$$

where $\Lambda_{e P}$ is the mass scale of a new pseudoscalar interaction in $\pi \rightarrow e \nu$ decay. This property makes the measurement of the branching ratio at a $0.1 \%$ level sensitive to the mass scales of $O(1000 \mathrm{TeV})$ for pseudoscalar interactions.

Scalar couplings arising from physics beyond the SM (i.e. scalar couplings which don't follow the SM Higgs mass dependence) will also induce pseudoscalar interactions through loop corrections and, in many cases, the $\pi \rightarrow e \nu$ branching ratio measurement provides substantially stronger limits than those from $\beta$-decay measurements [6].

Stronger limits on the existence of massive neutrinos in the mass region $\leq 40 \mathrm{MeV} / \mathrm{c}^{2}$ can also be set with improved statistics on the $\pi \rightarrow e \nu$ decay. Candidate examples of the new physics probed include the R-parity violating SUSY [7], effects of high scale four-fermion operators due to excited gauge bosons (e.g. from extra dimensions) [8], leptoquarks [9], compositeness [10] or charged Higgs bosons [7].

\section{The PIENU Experiment}

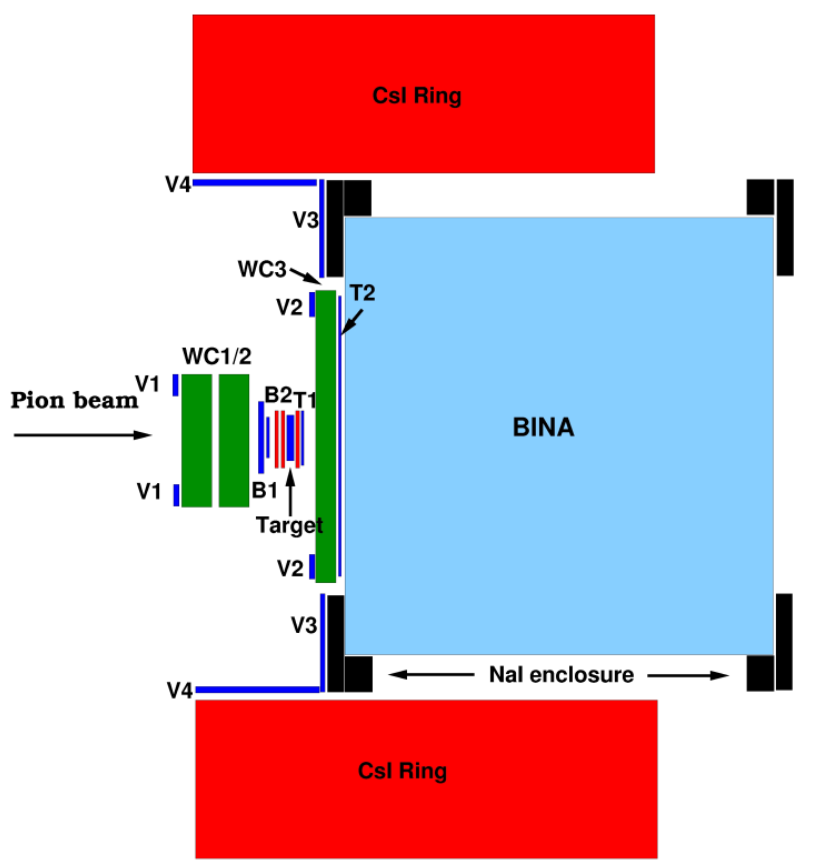

Figure 1. Experimental setup. 
Figure 1 shows the detector arrangement. A $75-\mathrm{MeV} / \mathrm{c} \pi^{+}$beam from the TRIUMF M13 channel with an intensity of $50-100 \mathrm{kHz}$ is identified by two beam counters and stopped in an active scintillator target. Beam tracking is provided by two wire chambers, and two silicon-strip counters located immediately upstream of the target.

Positrons from the decays $\pi^{+} \rightarrow \mathrm{e}^{+} \nu$ and $\pi^{+} \rightarrow \mu^{+} \nu$ followed by $\mu^{+} \rightarrow \mathrm{e}^{+} \nu \bar{\nu}$ decay $\left(\pi^{+} \rightarrow \mu^{+} \rightarrow \mathrm{e}^{+}\right.$decay) are measured in the positron telescope. It consists of a silicon-strip counter, two thin plastic counters, a third acceptance-defining wire chamber covering the front of a 48-cm-diameter, 48-cm-long single $\mathrm{NaI}(\mathrm{Tl})$, which provides the primary energy measurement. The solid angle of the telescope counters is $20 \%$. Two layers of 8.5 -cm-thick, $2 \times 25$-cm-long pure CsI counters surround the $\mathrm{NaI}$ to capture shower leakage. Analog signals from plastic scintillators (Si-strip, NaI and CsI detectors) are recorded by $500 \mathrm{MHz}$ Copper [11] (60 MHz VF48 [12]).

\section{The Measurement technique}

Simultaneous fitting of the time distributions, Fig.2, of low-energy $(\mathrm{E}<55 \mathrm{MeV})$ and high-energy $(\mathrm{E}>55 \mathrm{MeV}$ ) regions provides the yields of $\pi \rightarrow \mu \rightarrow e$ and $\pi \rightarrow e \nu$ decays, respectively.
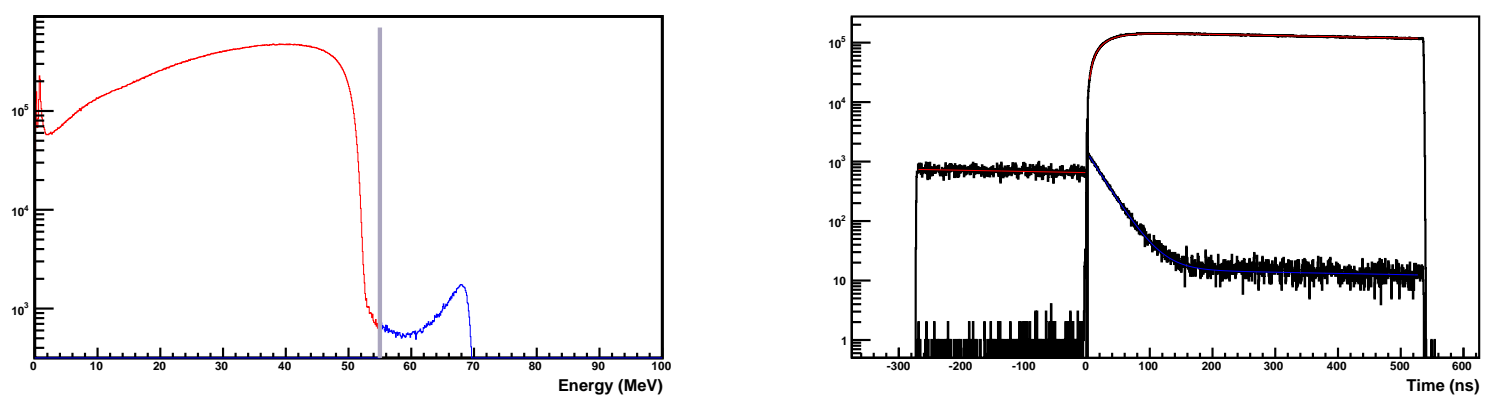

Figure 2. left : Energy spectrum. The grey line shows the energy between $\pi \rightarrow \mathrm{e} \nu$ (high energy, blue) and $\pi \rightarrow \mu \rightarrow$ e (low energy, red) decays . right: Fitted time spectra of both energy regions.

The dominant uncertainty in the previous TRIUMF experiment [1] was in the knowledge of the low-energy tail of $\pi \rightarrow \mathrm{e} \nu$ events in the energy region of the $\pi \rightarrow \mu \rightarrow$ e population. The same technique employed in [1] will be used to deduce the low-energy tail correction based on the positron energy spectrum in which the $\pi \rightarrow \mu \rightarrow$ e background is suppressed by selecting a small time window after the pion stop and using pulse shape and energy information in the target, Fig.3.

The majority of remaining low-energy events in the background-suppressed spectrum come from in-flight decays of pions around the target. Pion tracking near the target provides an additional background suppression. An overall improvement factor of $>5$ including improved statistics is expected for this correction. With statistics of $>30$ times those of the previous experiments and substantially reduced systematic uncertainties, the expected precision of the PIENU experiment for the branching ratio of the pion decays will be $<0.1 \%$, which corresponds to $<0.05 \%$ uncertainty in the ratio of the coupling constants $g_{e} / g_{\mu}$.

\section{Beam and detectors performances}

A high- purity pion beam is produced with an energy-loss separation technique in the TRIUMF M13 beam channel [13]. The high suppression of positron achieved (positron content $\leq 2 \%$ ) 

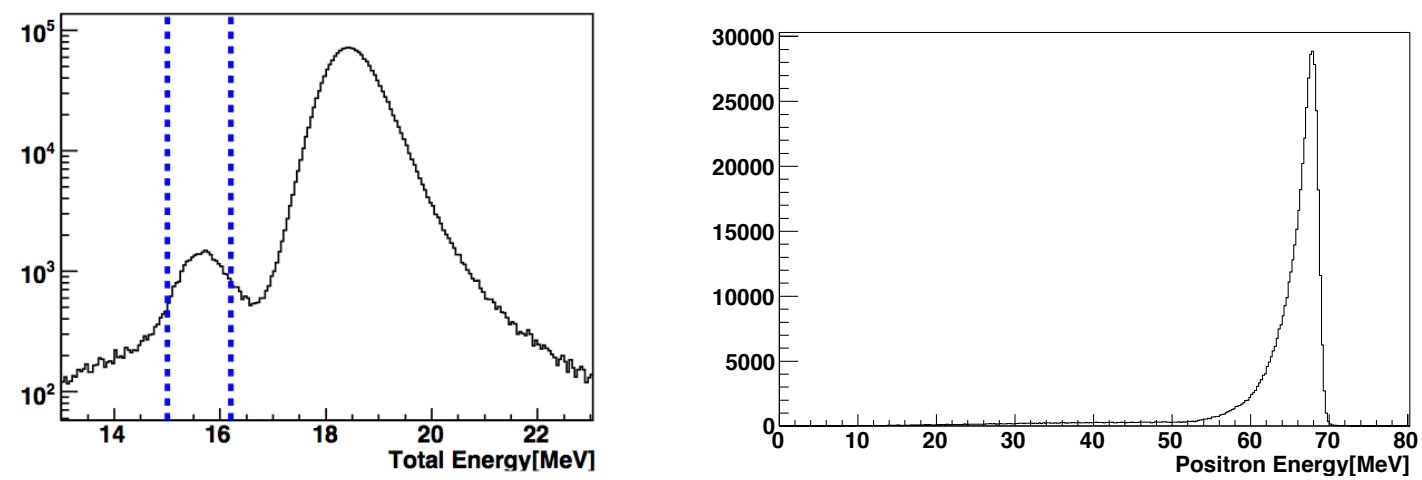

Figure 3. left : Energy deposit in the Target. The blue dotted lines show where the $\pi \rightarrow \mathrm{e} \nu$ decays are mainly concentrated. right : $\pi \rightarrow \mathrm{e} \nu$ spectrum obtained after suppression of in-flight decays.

with this technique reduces the impact of the trigger rate and the background on the $\pi \rightarrow \mathrm{e} \nu$ time spectrum.

Detailed knowledge of the crystal response is essential to determining the low energy tail response below $55 \mathrm{MeV}$. The response function of the NaI/CsI system was measured using 50-85 $\mathrm{MeV} / \mathrm{c}$ positron beams. The energy resolution was $2 \%$ (FWHM) including the beam contribution. The low energy tail for beam entrance angles of $0-40^{\circ}$, due mostly to shower leakage escaping the CsI crystals, was $0.5-1.5 \%$, consistent with Monte Carlo simulations as illustrated in Fig.4

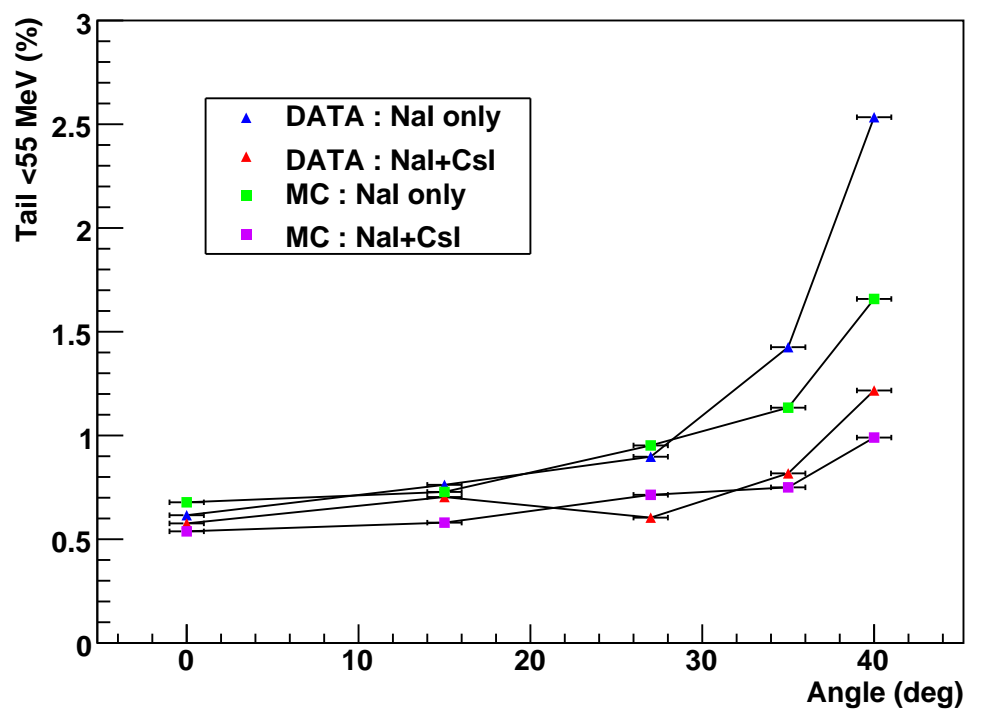

Figure 4. Fraction of events below $55 \mathrm{MeV} / \mathrm{c}$ vs. the angle of incidence on the face of the $\mathrm{NaI}$ crystal. Using energy measured in the CsI ring surrounding the $\mathrm{NaI}$ crystal brings the low energy tail below $1.5 \%$ for all measured $\mathrm{e}^{+}$entrance angles. 
A thorough study of the NaI calorimeter's response spectrum with beam positrons showed the existence of two low energy structures at around 9 and $17 \mathrm{MeV}$ below the main positron peak, Fig.5. Their origin was found to be consistent with neutron emission due to photo- absorption followed by neutron escape from the crystal [14].

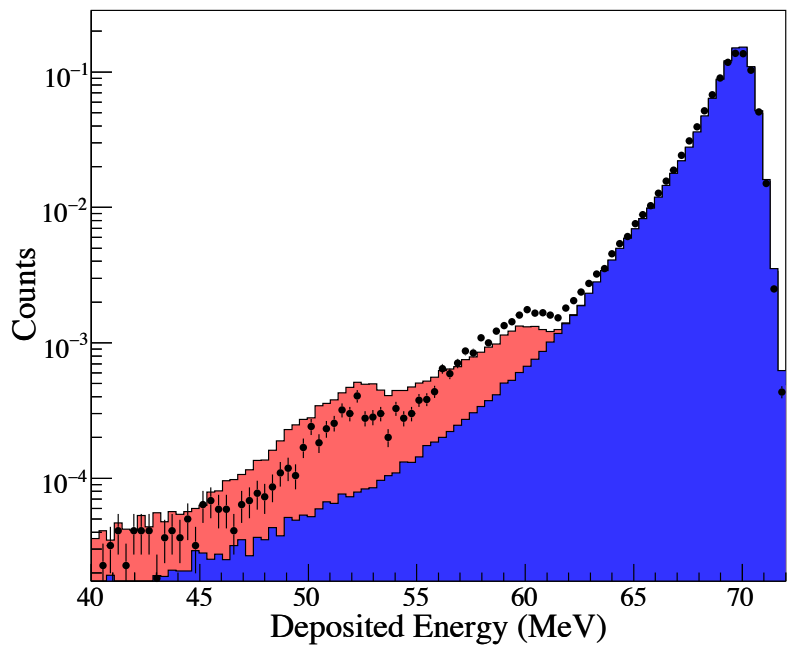

Figure 5. Comparison between data (filled circles with error bars) and simulation (coloured area). The simulation was performed with (red) and without (blue) hadronic reaction contributions. The histograms are normalized to the same area.

\section{Conclusion}

The PIENU experiment is currently in the second phase of physics data taking. Beamline and detectors have been tested and satisfy the requirements to achieve the goal of measuring the pion decay's branching ratio : $\frac{\Gamma\left(\pi^{+} \rightarrow e^{+} \nu_{e}+\pi^{+} \rightarrow e^{+} \nu_{e} \gamma\right)}{\Gamma\left(\pi^{+} \rightarrow \mu^{+} \nu_{\mu}+\pi^{+} \rightarrow \mu^{+} \nu_{\mu} \gamma\right)}$, to an accuracy five times better than the current world average value.

\section{References}

[1] D.I.Britton et al., Phys. Rev. Lett. 68, 3000 (1992) and Phys. Rev. D 49, 28 (1994).

[2] G. Czapek et al., Phys. Rev. Lett. 70, 17 (1993).

[3] W.J. Marciano and A. Sirlin, Phys. Rev. Lett. 71, 3629 (1993).

[4] V. Cirigliano and I. Rosell, JHEP 0710, 005 (2007).

[5] O. Shanker, Nucl. Phys. B 204:375 (1982).

[6] B. A. Campbell and D. W. Mayberry, Nucl. Phys. B 709, 419 (2005).

[7] M.J. Ramsey-Musolf, S. Su and S. Tulin, Phys. Rev. D 76, 095017 (2007).

[8] J. Maalampi et al., Phys. Rev. D 67,113005 (2003).

[9] M. Hirsch et al., Phys. Lett. B 378, 17 (1996).

[10] Nima Arkani-Hamed, Savas Dimopoulos, and G. R. Dvali, Phys. Lett., B429:263 272 (1998).

[11] K. Yamada et al., Nuclear Science, IEEE Transactions on Volume 54, Issue 4, Aug. 2007 Page(s):1222 - 1226 Digital Object Identifier 10.1109/TNS.2007.901220

[12] J.-P. Martin and P.-A. Amaudruz, Nuclear Science, IEEE Transactions on, Volume 53, Issue 3, Part 1, June 2006 Page(s):715 - 719 Digital Object Identifier 10.1109/TNS.2006.875049

[13] A.Aguilar-Arevalo et al., Nucl. Instr. and Meth. A 609, 102 (2009).

[14] A.Aguilar-Arevalo et al., Nucl. Instr. and Meth. A 621 (2010) 188191 